\title{
Flexible and Reconfigurable Layouts in Complex Manufacturing Systems
}

\author{
Maria Manuela Azevedo ${ }^{1,2, *}$, José António Crispim ${ }^{2,3}$, and Jorge Pinho de Sousa ${ }^{1,2}$ \\ ${ }^{1}$ Faculty of Engineering, University of Porto, Porto, Portugal \\ manuela.azevedo@fe.up.pt \\ ${ }^{2}$ INESC TEC, Porto, Portugal \\ ${ }^{3}$ School of Economics and Management, University of Minho, Braga, Portugal
}

\begin{abstract}
This paper studies the Facility Layout Problem (FLP) of a first tier supplier in the automotive industry. This complex manufacturing system involves multiple facilities, complex products, and layout reconfiguration constraints. One of the key requirements of this particular system is the need for high levels of flexibility in the reconfiguration of the layouts. This problem is formulated as a mixed-integer programming (MIP), based on a FLP model with multiple objectives and unequal areas. The model allows for two reconfiguration types: small and large changes. We explore the application of optimization methodologies to produce efficient and flexible layouts.
\end{abstract}

Keywords: Facility layouts, re-layouts, flexibility, optimization.

\section{Introduction}

The configuration of facility layouts involves the physical organization (of departments, machines, workstations, storage spaces, etc.) inside a plant, facilitating production and material handling, and allowing flexible and efficient operations. The expression "facility layout" is used here in a broad sense since our work is closely related to the literature both on facility location and supply chain design. Due to its practical importance to manufacturing systems competitiveness, this area has attracted a lot of interest from researchers. The reduction of product life cycles and the need to respond rapidly to market changes increase the importance of designing layouts that are more flexible, modular and easily reconfigurable [1]. In the automotive industry, product life cycles are relatively short and technological innovation plays an important role, making it still more important to incorporate the resulting dynamic features into the design process. However the extensive use of databases and benchmark data to test proposed methods often requires the simplification of the characteristics of real production and logistic systems, thus compromising their practical applicability. Moreover this industry produces a large variety of complex products (with many components) and services [2] that significantly increases production and flows complexity.

\footnotetext{
* Corresponding author.
} 
In this work we propose an optimization approach where the facility layout problem is formulated using mixed-integer programming (MIP). Unequal area FLPs are a class of extremely difficult and broad optimization problems arising in many diverse areas [3]. Unfortunately, most of these approaches are based on a single objective while real-world FLPs are naturally multi-objective.

The approach proposed here is meant to tackle FLPs with multiple objectives and unequal areas, as a way to respond to real-world requirements. The model is based on the combination of two sub-models. The first defines the relative position of the departments inside the facilities, and the second defines the relative position of machines inside departments and creates the necessary flows to determine the final layout. The data used to assess and validate the approach was collected from a case study in the automotive industry.

In the next section a brief literature review about facility layouts is presented. Section 3 defines the problem, Section 4 describes our general approach, and Section 5 presents some initial MIP models. Finally in Section 6 we present the preliminary conclusions of this research.

\section{Literature Review}

The Facility Layout Problem (FLP) has been extensively studied and, accordingly, there are numerous related research surveys in the literature ([4], [1] and [5] are some recent examples). There are essentially three types of approaches to solve FLPs (see [4] and[5]): i) optimization: finding optimal layouts; ii) heuristics: finding nearly optimal solutions with hopefully efficient procedures, and iii) simulation: providing a way to assess alternative, potentially interesting solutions. Procedures based on the combination of these different types have also been proposed.

As far as we are aware, there are no studies that focus on layout design involving all the production and storage facilities of a company and only a few studies exhibit detailed and flexible design layouts. For example, Krishnan et al. [6] describe a FLP approach to deal with the uncertainty of product demand in the design of a facility layout, for single-period and multi-period problems. The main drawback of this work is the assumption that all the departments have the same area. González-Cruz and Martínez [7] propose a new multi-criteria entropy-based algorithm for the generation and evaluation of different layout designs of workstations or departments in an industrial plant. However, they do not detail the layout design inside each workstation or department.

Other authors try to further detail the layout design. For example, Kia et al. [8] propose a model for the layout design of a dynamic cellular manufacturing system with product mix and part demands varying during a multi-period planning horizon. They make use of multi-rows layouts to locate machines in the cells configured with flexible shapes. This approach is restricted to cells of production departments. Dong et al. [9] studied the problem of added or removed machines in a plant but with the assumption that the list of machines is known in each period. 
The main innovative contribution of our work is that we do not simply focus on machine allocation or department configuration. Instead, we combine in a single approach, the problems in a manufacturing system, associated with machine allocation, department location and flows design. And to design this approach we have taken into account a set of general requirements directly derived from the real problematic situations of the case study.

According to Arabani and Farahani [10] more efforts should be made to incorporate multiple facilities in the analysis of facility location problems in order to effectively handle fluctuating demands originated from miscellaneous customers, industrial sectors and companies. Handling several facilities obviously increases the problem complexity, thus justifying the research presented in this work.

\section{The Problem}

Our problem consists in finding the best physical organization of facilities (departments, machines, workstations, warehouses, etc.) and the best flows of products and raw materials, fostering flexible and efficient operations (see Figure 1). A layout is efficient if the materials flow in a short and rapid way, without waste of time and resources, and it is flexible when it allows fast, cheap and easy to do reconfigurations.

In this work we consider a factory with physically distributed departments (e.g., production, assembly, warehouses, among others). Inside the departments we can have machines, workstations, storage areas and paths that connect them.

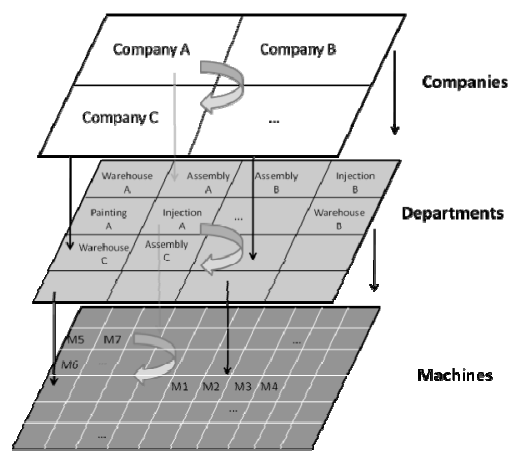

Fig. 1. Flows Complexity

In our real-world situation, there is a group of geographically separated facilities ( 3 factories with 3 warehouses and other production departments) that can produce and store the same type of products and components. Each facility has more or less the same department structure, with the same type of equipment and machines. These facilities are served by a distribution system with trucks to move the raw materials, components and/or products among them. Currently, each facility has warehouses that store the same raw materials, components and products since the production process is common to the factories. 
The company works with "projects" that are associated to specific models of a car. A project comprises several parts that are themselves composed by a group of materials or components (see Table 1). These can be produced or assembled by any factory of the group. Each project is assigned to one facility.

Table 1. Product Complexty (example)

\begin{tabular}{|c|c|c|c|c|c|c|c|c|}
\hline \multicolumn{9}{|c|}{ Project 1 } \\
\hline \multicolumn{3}{|c|}{ Part A } & Part B & \multicolumn{5}{c|}{ Part C } \\
\hline $\begin{array}{c}\text { Comp } \\
\text { I }\end{array}$ & $\begin{array}{c}\text { Comp } \\
\text { II }\end{array}$ & $\begin{array}{c}\text { Comp } \\
\text { III }\end{array}$ & $\begin{array}{c}\text { Comp } \\
\text { I }\end{array}$ & $\begin{array}{c}\text { Comp } \\
\text { I }\end{array}$ & $\begin{array}{c}\text { Comp } \\
\text { II }\end{array}$ & $\begin{array}{c}\text { Comp } \\
\text { III }\end{array}$ & $\begin{array}{c}\text { Comp } \\
\text { IV }\end{array}$ & $\begin{array}{c}\text { Comp } \\
\text { V }\end{array}$ \\
\hline
\end{tabular}

One of the main contributions of this research is the design of a decision-making procedure based on the concepts of "small" and "large" layout changes, making the manufacturing system more dynamic and flexible (see Figure 2).
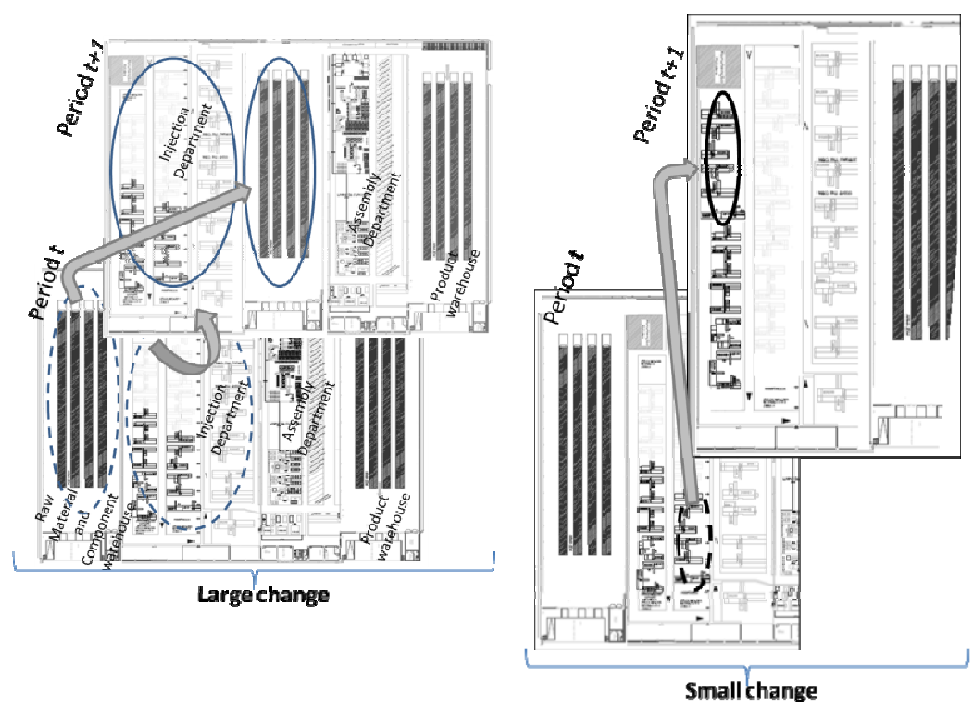

Fig. 2. Large and Small changes in a layout (example)

\section{The Approach}

Given its characteristics, this is a rather complex, NP-hard problem [5]. As Arabani and Farahani [10] point out, the main parameters of dynamic problems may significantly change over the planning time horizon, this implying we need to explicitly consider robustness and reliability criteria. Therefore, the models and methods developed to solve these problems should incorporate performance measures or objectives that take risk into account. 
The proposed approach (see Figure 3) is a dynamic FLP with multiple objectives and unequal areas, and it is based on the combination of two sub-models: the first defines the relative position of the departments inside various facilities, and the second determines the detailed layout, with the definition of machine positions, inside departments and the associated physical flows.

Our model allows for two re-configuration types: small and large changes. Large changes are required when departments need to be moved from one facility to another or change their position in the same facility, possibly as a result of the arrival of new projects. Small changes are more frequent and consist of reconfigurations inside a department by adding / dropping machines, or by redirecting the flows of materials and products in progress.

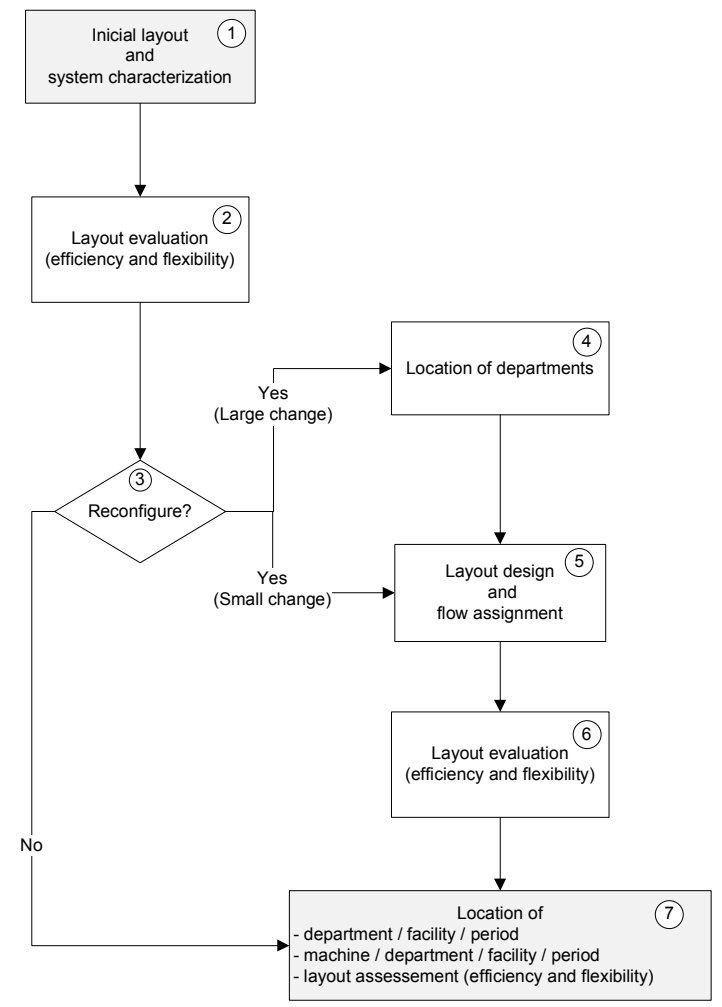

Fig. 3. Proposed approach

As presented in Figure 3 this approach consists of 7 steps. In step 1, one makes the system characterization, with parameter values and data. Then in step 2 , that information is evaluated, for the multilevel layout of facilities and their departments, in terms of use of equipment and of material flows. These results will be compared to predefined layout efficiency and flexibility targets, in step 3. If the levels of efficiency are satisfactory, the current layout is not changed. 
Otherwise, depending on the level of achieved efficiency, the required reconfiguration of the layout can be classified as "large" or "small". For example, if only one department has a low efficiency, only this department needs to be reconfigured, this consisting in a small change (step 5). On the other end, if the whole system has a low efficiency, the reconfiguration is considered to be large (step 4).

In step 4, new locations for the departments are determined, trying to minimize total costs. After changing the positions of the departments, it is necessary to internally reconfigure those departments, and this is done in step 5, by finding the position of machines inside each department and the respective flow assignment, in step 6, the new configuration is evaluated, in terms of efficiency and flexibility. Finally a complete solution is presented (step 7), with the layout configuration of the system, organized in different levels (facilities, departments), as well as the assignment of products to the different machines.

Given the complexity of the problem under analysis and the set of potentially interesting requirements, this approach is intended to be used to analyze different scenarios, namely:

- $\quad$ to centralize the warehouses in one facility;

- $\quad$ to combine departments of the same type in the same facility;

- $\quad$ to produce each product in a specific factory;

- $\quad$ to allow all factories to produce the same products.

The results of this type of analysis will hopefully lead to interesting, valuable guidelines for supporting strategic / tactical decision-making in the company.

\section{$5 \quad$ Mathematical Models}

Considering the complexity and features of the problem characterized by the case study, we have designed the mathematical models presented in this section. For computational purposes, these models are based on some simplifying assumptions and are formulated as mixed-integer programming, based on a Dynamic Facility Layout Problem with multiple objectives and unequal areas.

\subsection{General Concepts and Notation}

The following general assumptions have been considered:

- the cost of moving machines is known, but it depends on the type of machine and on the type of department;

- the distances between locations are computed between the physical centers of the locations, and measured with a rectilinear distance norm;

- a machine transforms an input into an output (a product), in reality - it can also be a workstation, that assemblies a product or perform other kind of function;

- all departments and machines can be moved to any location inside the facility or to other facility. 


\section{Indices}

$t=1,2, \ldots$ - periods of time

$f=1,2, \ldots$ - facilities

$i, j=1,2, \ldots$ - departments

$l, k=1,2, \ldots$ - positions (locations) in a facility or in a department

\section{General Parameters}

$a_{f}$ - area of facility $\mathrm{f}$

$a_{i}$ - area of department $\mathrm{i}$

$r_{l k f}$ - distance between position $\mathrm{l}$ and position $\mathrm{k}$, in facility $\mathrm{f}$

\subsection{Location of Departments ("large changes")}

This model makes the allocation of departments to places or positions at facilities, each period of time. This allocation could be maintained the same position from period to the next or change the position in the same facility or even change to other facility.

\section{Additional parameters}

$c r_{i}$ - fixed cost of shifting department $\mathrm{i}$

$q_{i j t}$ - flow (product quantity) between department $\mathrm{i}$ and $\mathrm{j}$, in period $\mathrm{t}$

Decision variables

$x_{\text {ilft }}=\left\{\begin{array}{c}1 \text { if department } i \text { is placed at position l, in facility } f, \text { in period } t \\ 0 \text { otherwise }\end{array}\right.$

Model

$\operatorname{Min} C 1=$

$\sum_{t, f, i, j, l, k}\left[q_{i j t} r_{l k f}\left(x_{i l f t} x_{j k f t}\right)\right]+\sum_{t, i, j, k, l, f 1, f 2}\left[c r_{i} r_{l f 1 k f 2}\left(x_{i l f 1 t} x_{j k f 1 t+1}\right)\right]$

subject to:

$$
\begin{aligned}
\sum_{i, l} a_{i f} x_{i l f t} \leq a_{f} \quad \forall f, t \\
\sum_{i} x_{i l f t} \leq 1 \quad \forall l, f, t \\
\sum_{l} x_{i l f t} \leq 1 \quad \forall i, f, t
\end{aligned}
$$

This model aims at minimizing total costs. The first term in the objective function (1) is related to the flows between departments and facilities, representing the material handling costs. The second term represents the reconfiguration cost, incurred when a department changes its position inside a facility or to another facility.

Constraints (2) ensure that the total area of a facility is never exceeded. Constraints (3) guarantee that a position in a facility has never more than one department, in each time period. Constraints (4) guarantee that a department is only assigned to one position in a facility, in each time period. 


\subsection{Layout Design and Flow Assignment ("Small Changes")}

This model is used to support the design of the detailed layout, determining the positions for the machines inside departments and assigning flows, i.e., allocating the different products to the different machines, in each time period.

\section{Other indices}

$\mathrm{m}, \mathrm{n}=1,2, \ldots$ - machines

$\mathrm{p}=1,2, \ldots-$ type of products

\section{Additional parameters}

$c r_{m}$ - fixed cost of shifting machine $m$

$c m_{m p}$ - capacity of machine $m$ when producing product $p$

$q_{m n t}$ - flow (product quantity) between machine $m$ and $n$, in period $t$

$a_{m}$ - area occupied by machine $m$

$r_{l k i}$ - distance between position $l$ and position $k$, in department $i$

$d_{p t}$ - demand of product $p$, in period $t$

Decision variables

$y_{\text {mlit }}=\left\{\begin{array}{c}1 \text { if machine } m \text { is placed at position l, in department } i, \text { in period } t \\ 0 \text { otherwise }\end{array}\right.$

$u_{m p t}=\left\{\begin{array}{c}1 \text { if machine } m \text { is used to produce product } p \text {, in period } t \\ 0 \text { otherwise }\end{array}\right.$

$b_{p m t}$ - quantity of product $p$, to be produced in machine $m$, in period $t$

\section{Model}

$\min C 2=$

$\sum_{t, i, n, m, l, k}\left[q_{m n t} r_{l k i}\left(y_{m l i t} y_{n k i t}\right)\right]+$

$\sum_{t, i 1, i 2, k, l, m, n}\left[c r_{m} r_{l i 1 k i 2}\left(y_{n l i 1 t} y_{m k i 1 t+1}\right)\right]$

$$
\max E=\sum_{t, m, p, i}\left(b_{p m t} u_{m p i t}\right)
$$

Subject to

$$
\begin{gathered}
\sum_{m, l} a_{m i} y_{m l i t} \leq a_{i} \quad \forall i, t \\
\sum_{m} y_{m l i t} \leq 1 \quad \forall l, i, t \\
\sum_{l} y_{m l i t} \leq 1 \quad \forall m, i, t \\
b_{p m t} \leq c m_{m p} \quad \forall t, p, m \\
\sum_{f, i, m, p}\left(c m_{p} u_{m p t i f}\right) \geq \sum_{p} d_{p t} \quad \forall t
\end{gathered}
$$

This model aims both at minimizing the total cost (5) and at maximizing the layout efficiency (6), in the each period of time. The first objective function (5) is similar to expression (1), with a first part related to the flows between machines, representing the material handling costs inside departments, and the second term representing the 
reconfiguration costs, incurred when a machine changes its position inside a department. The second objective function (6) maximizes the efficiency of the layout, by increasing the number of machines being used in each period of time.

Constraints (7) ensure that the total area of a department is never exceeded. Constraints (8) guarantee there is no overlap of machines, in each time period. Constraints (9) guarantee that a machine is only assigned to one position in a department, each time period. Constraints (10) verify that the quantity of product assigned to a machine does not exceed the machine capacity. Finally constraints (11) guarantee that the total installed capacity is enough to produce the total demand.

\subsection{Preliminary Computational Results}

These models are now being implemented and tested with IBM ILOG CPLEX Optimizer Studio version 12.2. For the above objective functions, which are not linear, we are using standard reformulation techniques to linearize them.

Preliminary computational results using small, randomly generated instances, seem to be satisfactory, thus validating the approach. It should be noted that, although random, these instances have been designed based on our knowledge of the case study, and seem to reflect the main issues to be solved in practice.

\section{Conclusions}

This work presents some preliminary results of a research project based on a case study involving the reconfiguration of the manufacturing system of a first tier supplier in the automotive industry. This case study considers the entire manufacturing system with multiple facilities. We allow for two types of reconfiguration that differ in the deepness and frequency of the modifications (these alternatives are referred as "large" and "small" changes).

Accordingly the problem was partitioned in two components, and formulated using mixed-integer programming (MIP), based on a FLP model with multiple objectives and unequal areas. This seems to create a useful tool to support the design and reconfiguration of flexible layouts, allowing more efficient operations, in rather dynamic environments. A first assessment of the first results of this approach by the decision-makers has shown its potential, thus justifying further developments along this line.

Acknowledgments. The authors acknowledge the financial support from FCT, the Portuguese Foundation for Science and Technology (under grant SFRH/BD/33731/ 2009) and from the MIT Portugal Program.

\section{References}

1. Kulturel-Konak, S.: Approaches to uncertainties in facility layout problems: Perspectives at the beginning of the 21st Century. Journal of Intelligent Manufacturing 18, 273-284 (2007) 
2. Marengo, L., Valente, M.: Industry dynamics in complex product spaces: An evolutionary model. Structural Change and Economic Dynamics 21, 5-16 (2010)

3. Ripon, K.S.N., Khan, K.N., Glette, K., Hovin, M., Torresen, J.: Using pareto-optimality for solving multi-objective unequal area facility layout problem. In: Proceedings of the 13th Annual Conference on Genetic and Evolutionary Computation - GECCO 2011, p. 681. ACM Press, New York (2011)

4. Moslemipour, G., Lee, T.S., Rilling, D.: A review of intelligent approaches for designing dynamic and robust layouts in flexible manufacturing systems. The International Journal of Advanced Manufacturing Technology (2011)

5. Drira, A., Pierreval, H., Hajri-Gabouj, S.: Facility layout problems: A survey. Annual Reviews in Control 31, 255-267 (2007)

6. Krishnan, K.K., Jithavech, I., Liao, H.: Mitigation of risk in facility layout design for single and multi-period problems. International Journal of Production Research 47, 5911-5940 (2009)

7. González-Cruz, M.C., Gómez-Senent Martínez, E.: An entropy-based algorithm to solve the facility layout design problem. Robotics and Computer-Integrated Manufacturing 27, 88-100 (2011)

8. Kia, R., Baboli, A., Javadian, N., Tavakkoli-Moghaddam, R., Kazemi, M., Khorrami, J.: Solving a group layout design model of a dynamic cellular manufacturing system with alternative process routings, lot splitting and flexible reconfiguration by simulated annealing. Computers \& Operations Research, 1-17 (2012)

9. Dong, M., Wu, C., Hou, F.: Shortest path based simulated annealing algorithm for dynamic facility layout problem under dynamic business environment. Expert Systems with Applications 36, 11221-11232 (2009)

10. Arabani, A.B., Farahani, R.Z.: Facility location dynamics: An overview of classifications and applications. Computers \& Industrial Engineering 62, 408-420 (2012) 